\title{
molecules
}

ISSN 1420-3049

www.mdpi.com/journal/molecules

Article

\section{Quinoline-3-carboxamide Derivatives as Potential Cholesteryl Ester Transfer Protein Inhibitors}

Wen-Yan Li ${ }^{1}$, Xu-Qiong Xiong ${ }^{1}$, Dong-Mei Zhao ${ }^{1, *}$, Yu-Fang Shi ${ }^{2}$, Zhi-Heng Yang ${ }^{1}$, Chao Yu ${ }^{1}$, Pei-Wei Fan ${ }^{1}$, Mao-Sheng Cheng ${ }^{1}$ and Jing-Kang Shen ${ }^{2, *}$

1 Key Laboratory of Structure-Based Drugs Design \& Discovery of Ministry of Education, School of Pharmaceutical Engineering, Shenyang Pharmaceutical University, Shenyang 110016, China

2

Central Research Institute, Shanghai Pharmaceuticals Holding Co., Ltd., Shanghai 201203, China

* Authors to whom correspondence should be addressed; E-Mails: dongmeiz-67@163.com (D.-M.Z.); jkshen@mail.shcnc.ac.cn (J.-K.S.); Tel.: +86-024-2398-6413 (D.-M.Z.);

Fax: +86-024-2399-5043 (D.-M.Z.); Tel.: +86-021-5080-6600 (J.-K.S.);

Fax: +86-021-5080-7088 (J.-K.S.).

Received: 26 March 2012; in revised form: 14 April 2012 / Accepted: 20 April 2012 /

Published: 9 May 2012

\begin{abstract}
A series of novel quinoline-3-carboxamide derivatives 10-17 and 23-27 were designed and synthesized as cholesteryl ester transfer protein (CETP) inhibitors. All of them exhibited activity against CETP. Particularly, compounds $\mathbf{2 4}$ and $\mathbf{2 6}$ displayed the best activity against CETP with the same inhibitory rate of $80.1 \%$.
\end{abstract}

Keywords: CETP inhibitors; CETP; quinoline-3-carboxamide derivatives; synthesis; high density lipoprotein cholesterol

\section{Introduction}

Coronary heart disease (CHD), a leading cause of death around the World [1], has an inverse relationship with serum high density lipoprotein (HDL) cholesterol levels [2]. Raising of HDL cholesterol levels provides a potential therapeutic benefit for CHD patients [3,4]. Cholesteryl ester transfer protein (CETP) is a plasma glycoprotein which plays an important role in decreasing HDL-C level and increasing LDL-C level [5]. Inhibition of CETP may be a new therapy for atherosclerosis [6-10]. The research on CETP inhibitors such as dalcetrapib, anacetrapib and evacetrapib (Figure 1) has become a new hotspot for the treatment of CHD [11-13]. 
Figure 1. Structures of current CETP inhibitors.<smiles>CCC(CC)CC1(C(=O)Nc2ccccc2SC(=O)C(C)C)CCCCC1</smiles>

Dalcetrapib (Racho \& Tobacoo) Phase III clinical trial<smiles>COc1cc(F)c(C(C)C)cc1-c1ccc(C(F)(F)F)cc1CN1C(=O)O[C@@H](c2cc(C(F)(F)F)cc(C(F)(F)F)c2)C1C</smiles>

Anacetrapib (Merck) Phase III clinical trial<smiles>Cc1cc(C)c2c(c1)[C@@H](N(Cc1cc(C(F)(F)F)cc(C(F)(F)F)c1)c1nnn(C)n1)CCCN2C[C@H]1CC[C@H](C(=O)O)CC1</smiles>

Evacetrapib (Lily) Phase II clinical trial

In our lab a series of 2,3-dihydro-4-tetrahydroquinolones were found to have potent CETP inhibitory activity by virtual screening, including lead-like rule, clustering analysis, biological activity spectra prediction, ADME/Tox prediction and synthetic feasibility prediction [14]. Compound $\mathbf{1}$, a best effective CETP inhibitor among these derivatives, showed $30 \%$ inhibitory rate against CETP at $10 \mu \mathrm{M}$ in a buffer assay. In this study, our primary objective was to optimize the potency of compound $\mathbf{1}$ against CETP and obtain more potent CETP inhibitors. Based on the scaffold hopping strategy, 2,3-dihydro-4-tetrahydroquinolone was changed to a quinoline ring while retaining the aryl group at the 6 position (Figure 2). 6-Arylquinoline-3-carboxamide derivatives 10-17 were thus designed and synthesized. The crystal structure of CETP reveals a long continuous tunnel $(60 \AA)$ with a very large volume $\left(2,560 \AA^{3}\right)[15]$, so in order to make the structures bind nicely with CETP in the bulky active cavity, the phenyl ring substituent was replaced by larger and more flexible 6-benzyloxy and 7-methoxy groups to give the derivatives 23-27 which were also synthesized. All of target compounds 10-17, 23-27 were evaluated their CETP inhibitory activity by a radioisotope-based assay.

Figure 2. Scaffold hopping from tetrahydroquinolone into quinoline analogues.<smiles>CCOC(=O)c1cc(-c2ccccc2)cc2c1NCC(CN1CCCC1)C2=O</smiles><smiles>[R]NC(=O)c1cc2cc(-c3ccc([R])cc3)ccc2nc1C</smiles>

1 Inhibitory rate $=30 \%(10 \mathrm{uM})$<smiles>[R]NC(=O)c1cc2cc(OCc3ccccc3)c(OC)cc2nc1C</smiles> 


\section{Result and Discussion}

\subsection{Chemistry}

The synthetic pathway to the 6-phenylquinoline-3-carboxamide derivatives is shown in Scheme 1. Compound 3 was obtained in $65 \%$ yield by treatment of 3 -bromobenzaldehyde 2 with violet acid $\left(\mathrm{H}_{2} \mathrm{SO}_{4}: \mathrm{HNO}_{3}=10: 1, \mathrm{~V} / \mathrm{V}\right)$. Suzuki coupling of aryl bromide 3 with phenylboronic acid or 4-methylphenylboronic acid gave compounds $\mathbf{4}$ or $\mathbf{5}$, which were reduced with zinc dust to provide substituted $o$-amino benzaldehydes 6 or 7. 3-Quinolinecarboxylic acids 8 or 9 was prepared by cyclization of 6 or 7 with ethyl acetoacetate and $p$-tolylsulfonic acid at $100{ }^{\circ} \mathrm{C}$ and then hydrolyzed with $5 \% \mathrm{NaOH}$ solution. Coupling of $\mathbf{8}$ or $\mathbf{9}$ with commercially available amines afforded the target compounds 10-17.

Scheme 1. The synthesis of 6-phenylquinoline-3-carboxamide derivatives 10-17.

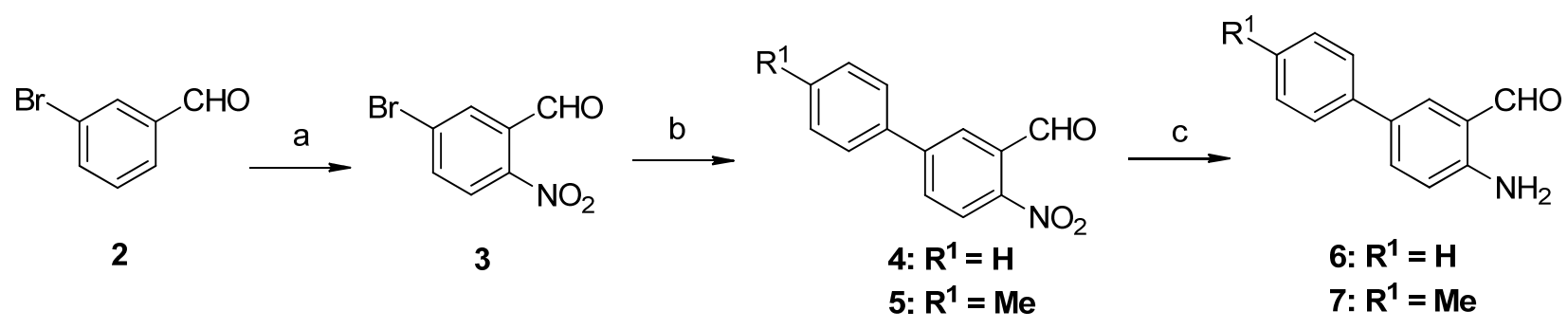

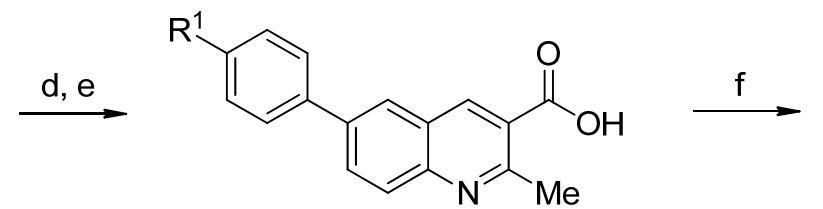

8: $\mathbf{R}^{1}=\mathbf{H}$

9: $R^{1}=M e$<smiles>[R]NC(=O)c1cc2cc(-c3ccc([R])cc3)ccc2nc1C</smiles>

10: $R^{1}=H, R^{2}=n$-decyl;

11: $R^{1}=H, R^{2}=$ benzyl ;

12: $R^{1}=H, R^{2}=$ cyclopropyl ;

13: $R^{1}=$ Me, $R^{2}=$ phenylethyl ;

14: $R^{1}=$ Me, $R^{2}=4$-methoxyphenyl ;

15: $\mathbf{R}^{1}=\mathrm{Me}, \mathbf{R}^{2}=$ benzyl ;

16: $\mathbf{R}^{1}=\mathrm{Me}, \mathbf{R}^{2}=$ cyclopropyl;

17: $\mathbf{R}^{\mathbf{1}}=\mathbf{M e}, \mathbf{R}^{\mathbf{2}}=\mathbf{t}$-butyl ;

Reagents and conditions: (a) $\mathrm{H}_{2} \mathrm{SO}_{4}, \mathrm{HNO}_{3},-5{ }^{\circ} \mathrm{C}$ to r.t., $0.5 \mathrm{~h}, 65 \%$; (b) $\mathrm{PhB}(\mathrm{OH})_{2}$ or 4- $\mathrm{MePhB}(\mathrm{OH})_{2}, \mathrm{Pd}(\mathrm{OAc})_{2}, \mathrm{CH}_{3} \mathrm{COCH}_{2} \mathrm{COMe}, \mathrm{K}_{2} \mathrm{CO}_{3}, \mathrm{EtOH}$, reflux, $1 \mathrm{~h}, 68 \%-72 \%$; (c) $\mathrm{Zn}$, $\mathrm{NH}_{4} \mathrm{Cl}$, EtOH, $\mathrm{H}_{2} \mathrm{O}$, r.t., 2 h, 79\%-84\%; (d) $\mathrm{CH}_{3} \mathrm{COCH}_{2} \mathrm{COOEt}, p$-TSA, $100{ }^{\circ} \mathrm{C}, 10 \mathrm{~min}$; (e) 5\% $\mathrm{NaOH}$, reflux, 2 h, 69\%-81\% (two steps); (f) $\mathrm{R}^{2} \mathrm{NH}_{2}$, HOBt, EDC.HCl, DIEA, DMF, r.t., 5 h, $22 \%-84 \%$.

The preparation of the 6-benzyloxy-7-methoxyquinoline-3-carboxamide derivatives is shown in Scheme 2. Alkylation of commercially available isovanillin (18) with benzyl bromide and potassium carbonate at $90{ }^{\circ} \mathrm{C}$ in DMF produced compound 19, which was nitrated with nitric acid at $0{ }^{\circ} \mathrm{C}$ to afford 20. The later steps were same as the synthesis of the 6-phenylquinoline-3-carboxamide derivatives. 
Scheme 2. The synthesis of 6-benzyloxy-7-methoxyquinoline-3-carboxamide derivatives 23-27.<smiles>COc1ccc(C=O)cc1OCc1ccccc1</smiles>

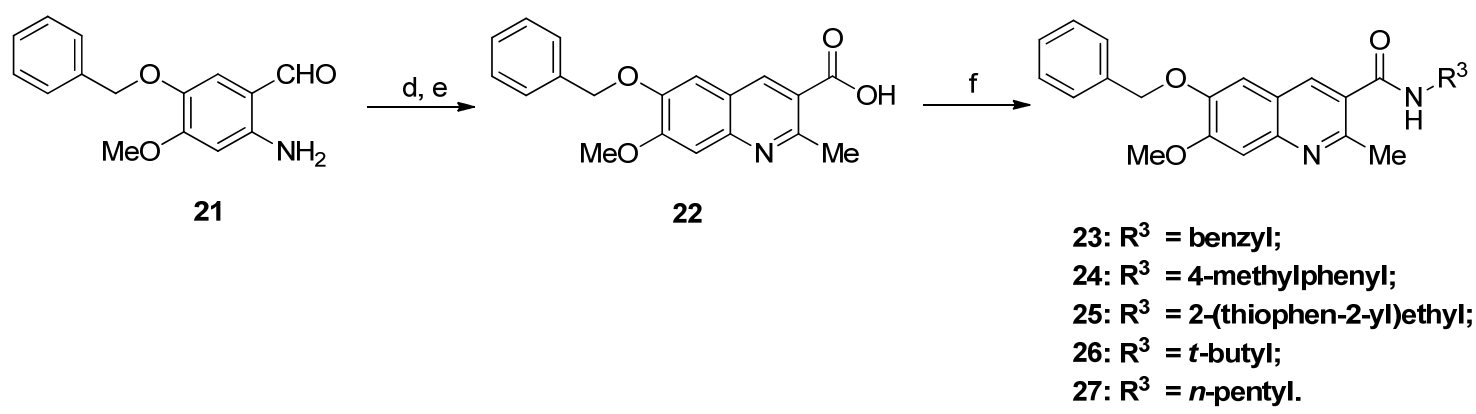

Reagents and conditions: (a) $\mathrm{BnBr}, \mathrm{K}_{2} \mathrm{CO}_{3}$, DMF, $90{ }^{\circ} \mathrm{C}, 3 \mathrm{~h}, 91 \%$; (b) $\mathrm{HNO}_{3}, 0{ }^{\circ} \mathrm{C}$ to r.t., $2 \mathrm{~h}, 78 \%$; (c) $\mathrm{Zn}, \mathrm{NH}_{4} \mathrm{Cl}$, EtOH, $\mathrm{H}_{2} \mathrm{O}$, r.t., 64\%; (d) $\mathrm{CH}_{3} \mathrm{COCH}_{2} \mathrm{COOEt}, p$-TSA, $100{ }^{\circ} \mathrm{C}, 10 \mathrm{~min}$; (e) $5 \%$ $\mathrm{NaOH}$, reflux, 5 h, $76 \%$ (two steps); (f) $\mathrm{R}^{3} \mathrm{NH}_{2}$, HOBt, EDC·HCl, DIEA, DMF, r.t., 5 h, $27 \%-73 \%$.

\subsection{Biological Activity}

In vitro inhibitory activity of all target compounds 10-17, 23-27 against CETP was evaluated by a radioisotope-based assay. The inhibition (\%) results are presented in Table 1. All the newly synthesized derivatives exhibited considerable CETP inhibitory activity (inhibitory rates: 20.7\%-80.1\%). Particularly, $p$-tolyl amide 24 and $t$-butyl substituted amide 26 exhibited the best inhibitory activity towards CETP, with the same inhibitory rate of $80.1 \%$, which is approximately the same activity of the positive control dalcetrapib. Substitution at 6 and 7 positions of the quinoline-3-carboxamide scaffold was considered a determining factor in the activity. Indeed, compounds 26, 24 and 23 with 6-benzyloxy-7-methoxy groups showed better activity than the corresponding substituted 6-phenyl analogues 17, 14 and 11, respectively. We speculate that the bulky 6-benzyloxy-7-methoxy group packs nicely in the large hydrophobic cavity of CETP. In addition, compounds 14 and 24 with a substituted aryl group on the 3-carboxamide nitrogen atom showed higher potency than other corresponding alkyl analogues in the two chemical classes.

Table 1. CETP inhibitory rate of the target compounds $(\mathbf{1 0}-\mathbf{1 7}, \mathbf{2 3}-\mathbf{2 7})$.

\begin{tabular}{cccc}
\hline Compd. & Inhibition (\%) $^{\text {a }}$ & Compd. & Inhibition (\%) $^{\mathbf{a}}$ \\
\hline $\mathbf{1 0}$ & $30.0 \pm 1.2$ & $\mathbf{1 7}$ & $35.2 \pm 0.7$ \\
$\mathbf{1 1}$ & $34.2 \pm 0.7$ & $\mathbf{2 3}$ & $51.5 \pm 2.6$ \\
$\mathbf{1 2}$ & $41.8 \pm 1.5$ & $\mathbf{2 4}$ & $80.1 \pm 1.3$ \\
$\mathbf{1 3}$ & $31.5 \pm 1.1$ & $\mathbf{2 5}$ & $29.8 \pm 2.5$ \\
$\mathbf{1 4}$ & $71.7 \pm 2.2$ & $\mathbf{2 6}$ & $80.1 \pm 0.8$ \\
$\mathbf{1 5}$ & $52.5 \pm 2.1$ & $\mathbf{2 7}$ & $20.7 \pm 2.3$ \\
$\mathbf{1 6}$ & $36.7 \pm 1.9$ & Dalcetrapib $^{\mathrm{b}}$ & $82.5 \pm 2.0$ \\
\hline
\end{tabular}

${ }^{\text {a }}$ Values represent the percent inhibition of CETP at $10 \mu \mathrm{M}$ of the test compounds and are the average of three independent experiments; ${ }^{b}$ The positive control. 


\section{Experimental}

\subsection{General}

All melting points were obtained on a Büchi Melting Point B-540 apparatus (Büchi Labortechnik, Flawil, Switzerland) and were uncorrected. Mass spectra (MS) were taken in ESI mode on Agilent 1100 LC-MS (Agilent, Palo Alto, CA, USA). Nuclear magnetic resonance spectroscopy was performed using Bruker ARX-300, $300 \mathrm{MHz}$ spectrometers (Bruker Bioscience, Billerica, MA, USA) with TMS as an internal standard. IR spectra ( $\mathrm{KBr}$ disks) were recorded with a Bruker IFS-55 instrument (Bruker). All the materials were obtained from commercially available sources and used without further purification, unless otherwise specified. Yields were not optimized. Compound 21 was synthesized according to the literature $[16,17]$.

5-Bromo-2-nitrobenzaldehyde (3). Compound 2 (27.1 g, $0.15 \mathrm{~mol})$ was slowly added dropwise to violet acid $\left(150 \mathrm{~mL}, \mathrm{H}_{2} \mathrm{SO}_{4}: \mathrm{HNO}_{3}=10: 1, \mathrm{~V} / \mathrm{V}\right)$ cooled to $-5^{\circ} \mathrm{C}$ and then stirred at room temperature for $0.5 \mathrm{~h}$. The reaction mixture was poured into ice water to give a white precipitate which was filtered off and purified by recrystallization from $5: 1$ petroleum ether/ethyl acetate to give the desired product $3(21.7 \mathrm{~g}, 65 \%)$ as a off-white solid; m.p. $60.5-61.9^{\circ} \mathrm{C} .{ }^{1} \mathrm{H}-\mathrm{NMR}\left(\mathrm{CDCl}_{3}\right) \delta: 7.89\left(1 \mathrm{H}, \mathrm{dd}, J_{l}=8.7 \mathrm{~Hz}\right.$, $\left.J_{2}=2.1 \mathrm{~Hz}\right), 8.03(1 \mathrm{H}, \mathrm{d}, J=8.7 \mathrm{~Hz}), 8.06(1 \mathrm{H}, \mathrm{d}, J=2.1 \mathrm{H}), 10.41(1 \mathrm{H}, \mathrm{s})$.

4-Nitrobiphenyl-3-benzaldehyde (4). To a solution of $3(22.7 \mathrm{~g}, 0.1 \mathrm{~mol})$ in alcohol $(150 \mathrm{~mL})$ and $\mathrm{K}_{2} \mathrm{CO}_{3}(1 \mathrm{M})(150 \mathrm{~mL})$ was added phenylboronic acid $(16.8 \mathrm{~g}, 0.14 \mathrm{~mol})$ and then $\mathrm{Pd}(\mathrm{AcO})_{2}(0.10 \mathrm{~g}$, $0.4 \mathrm{mmol})$ and acetylacetone $(0.3 \mathrm{~mL}, 1.2 \mathrm{mmol})$. The mixture was refluxed for $1 \mathrm{~h}$ and cooled to room temperature. The solution was concentrated and quenched with water, then extracted with ethyl acetate. The organic layer was washed with water and brine, and then dried over $\mathrm{Na}_{2} \mathrm{SO}_{4}$. Solvent was removed under reduced pressure and the resulting residue was purified by column chromatography (25\% EtOAc/petroleum ether, silica) to provide the title compound (16.3 g, 72\% yield) as a yellow solid; m.p. $71.0-73.4{ }^{\circ} \mathrm{C} .{ }^{1} \mathrm{H}-\mathrm{NMR}\left(\mathrm{CDCl}_{3}\right) \delta: 7.49(2 \mathrm{H}, \mathrm{t}, J=6.3 \mathrm{~Hz}), 7.50(1 \mathrm{H}, \mathrm{t}, J=6.3 \mathrm{~Hz}), 7.66$ $(2 \mathrm{H}, \mathrm{d}, J=8.1 \mathrm{~Hz}), 7.93(1 \mathrm{H}, \mathrm{d}, J=8.4 \mathrm{~Hz}), 8.1(1 \mathrm{H}, \mathrm{d}, J=2.1 \mathrm{~Hz}), 8.22(1 \mathrm{H}, \mathrm{d}, J=8.4 \mathrm{~Hz}), 10.51(1 \mathrm{H}, \mathrm{s})$.

4'-Methyl-4-nitrobiphenyl-3-benzaldehyde (5). 5 was obtained as a yellow solid (67.7\% yield) from compound 3 as described for 4; m.p. 54.2-58.4 ${ }^{\circ} \mathrm{C} .{ }^{1} \mathrm{H}-\mathrm{NMR}\left(\mathrm{CDCl}_{3}\right) \delta: 2.43(3 \mathrm{H}, \mathrm{s}), 7.32(2 \mathrm{H}, \mathrm{d}$, $J=8.1 \mathrm{~Hz}), 7.56(2 \mathrm{H}, \mathrm{d}, J=8.1 \mathrm{~Hz}), 7.92(1 \mathrm{H}, \mathrm{d}, J=8.4 \mathrm{~Hz}), 8.1(1 \mathrm{H}, \mathrm{d}, J=2.1 \mathrm{~Hz}), 8.20(1 \mathrm{H}, \mathrm{d}$, $J=8.4 \mathrm{~Hz}), 10.52(1 \mathrm{H}, \mathrm{s})$.

4-Aminobiphenyl-3-benzaldehyde (6). To a solution of $4(5.0 \mathrm{~g}, 22 \mathrm{mmol})$ in water $(100 \mathrm{~mL})$ and alcohol $(33 \mathrm{~mL})$ was added ammonium chloride $(7.1 \mathrm{~g}, 132 \mathrm{mmol})$ and then zinc dust $(17.2 \mathrm{~g}, 264$ $\mathrm{mmol})$. The mixture was stirred at room temperature for $2 \mathrm{~h}$ and filtered. The filtrate was concentrated and quenched with water, then extracted with ethyl acetate. The organic layer was washed with water and brine and then dried over $\mathrm{Na}_{2} \mathrm{SO}_{4}$. Solvent was removed under reduced pressure and obtained 6 (3.4 g, 79\% yield) as a yellow solid used directly to the next step without any purification; m.p. 124.5-125.8 ${ }^{\circ} \mathrm{C} .{ }^{1} \mathrm{H}-\mathrm{NMR}\left(\mathrm{CDCl}_{3}\right) \delta: 6.19(2 \mathrm{H}, \mathrm{s}), 6.77(1 \mathrm{H}, \mathrm{d}, J=8.5 \mathrm{~Hz}), 7.33(2 \mathrm{H}, \mathrm{t}, J=7.3 \mathrm{~Hz})$, $7.45(2 \mathrm{H}, \mathrm{t}, J=7.3 \mathrm{~Hz}), 7.56(1 \mathrm{H}, \mathrm{d}, J=7.1 \mathrm{~Hz}), 7.61\left(1 \mathrm{H}, \mathrm{dd}, J_{1}=2.2 \mathrm{~Hz}, J_{2}=8.5 \mathrm{~Hz}\right), 7.74(1 \mathrm{H}, \mathrm{d}$, $J=2.2 \mathrm{~Hz}), 9.98(1 \mathrm{H}, \mathrm{s})$. MS $m / z: 198.1[\mathrm{M}+\mathrm{H}]^{+}$. 
4'-Methyl-4-aminobiphenyl-3-benzaldehyde (7). Compound 7 was obtained from compound 5 as a yellow solid (84.8\% yield) as described for $\mathbf{6}$ and used directly to the next step without any purification; m.p. $148.2-149.4{ }^{\circ} \mathrm{C}$. MS $m / z: 212.1[\mathrm{M}+\mathrm{H}]^{+}$.

6-Phenyl-2-methylquinoline-3-carboxylic acid (8). Compound 6 (3.4 g, $17 \mathrm{mmol})$ was mixed with acetoacetic ester $(2.3 \mathrm{~g}, 17 \mathrm{mmol})$ and $p$-tolylsulfonic acid $(2.9 \mathrm{~g}, 17 \mathrm{mmol})$. The mixture was heated to $100{ }^{\circ} \mathrm{C}$ for $10 \mathrm{~min}$ and cooled to room temperature. Next $10 \% \mathrm{NaOH}(20 \mathrm{~mL})$ was added and stirred for $30 \mathrm{~min}$ to give a yellow precipitate. The precipitate was isolated by filtration and washed with water to give 8 as a yellow solid. Without any purification, the solid was dissolved in $5 \% \mathrm{NaOH}$ $(100 \mathrm{~mL})$. The solution was refluxed for $2 \mathrm{~h}$ and cooled to room temperature. The reaction mixture's $\mathrm{pH}$ value was adjusted to 2 with $6 \mathrm{~N}$ hydrochloric acid giving a greyish white precipitate. The precipitate was filtered and washed with water to $\mathrm{pH}$ 7. The filter cake was dried to give $\mathbf{8}$ (3.1 $\mathrm{g}, 69 \%$ yield for two steps) as a greyish white solid; m.p. 187.8-188.9 ${ }^{\circ} \mathrm{C} .{ }^{1} \mathrm{H}-\mathrm{NMR}$ (DMSO- $\left.d_{6}\right) \delta: 2.96(3 \mathrm{H}, \mathrm{s})$, $7.46(1 \mathrm{H}, \mathrm{t}, J=7.1 \mathrm{~Hz}), 7.55(2 \mathrm{H}, \mathrm{t}, J=7.2 \mathrm{~Hz}), 7.85(2 \mathrm{H}, \mathrm{d}, J=7.4 \mathrm{~Hz}), 8.30-8.19(2 \mathrm{H}, \mathrm{m}), 8.53(1 \mathrm{H}, \mathrm{d}$, $J=8.8 \mathrm{~Hz}), 9.08(1 \mathrm{H}, \mathrm{s}), 11.20(1 \mathrm{H}, \mathrm{s}) . \mathrm{MS} m / z: 262.1[\mathrm{M}-\mathrm{H}]^{-}$.

2-Methyl-6-(p-tolyl)quinoline-3-carboxylic acid (9). Compound 9 was obtained from compound 7 as a greyish white solid (80.9\% yield) as described for 8; m.p. 201.8-203.4 ${ }^{\circ} \mathrm{C} .{ }^{1} \mathrm{H}-\mathrm{NMR}\left(\mathrm{CDCl}_{3}\right) \delta: 2.45$ $(3 \mathrm{H}, \mathrm{s}), 2.96(3 \mathrm{H}, \mathrm{s}), 7.30(2 \mathrm{H}, \mathrm{d}, J=8.0 \mathrm{~Hz}), 7.46(2 \mathrm{H}, \mathrm{d}, J=7.9 \mathrm{~Hz}), 8.28-8.17(2 \mathrm{H}, \mathrm{m}), 8.49(1 \mathrm{H}, \mathrm{d}$, $J=8.8 \mathrm{~Hz}), 9.01(1 \mathrm{H}, \mathrm{s}), 11.1(1 \mathrm{H}, \mathrm{s})$.

3'-O-Benzylisovanillin (19). Compound 19 was obtained as a white solid (92\% yield) according to the literature [17]; m.p. 50.1-52.9 ${ }^{\circ} \mathrm{C} .{ }^{1} \mathrm{H}-\mathrm{NMR}\left(\mathrm{CDCl}_{3}\right) \delta: 3.96(3 \mathrm{H}, \mathrm{s}), 5.22(2 \mathrm{H}, \mathrm{s}), 7.01(1 \mathrm{H}, \mathrm{d}, J=8.1 \mathrm{~Hz})$, $7.25-7.49(7 \mathrm{H}, \mathrm{m}), 9.80(1 \mathrm{H}, \mathrm{s})$.

5-Benzyloxy-4-methoxy-2-nitrobenzaldehyde (20). Compound $\mathbf{2 0}$ was obtained as a white solid (78\% yield) according to the literature [16]; m.p. $131.8-133.0{ }^{\circ} \mathrm{C} .{ }^{1} \mathrm{H}-\mathrm{NMR}\left(\mathrm{CDCl}_{3}\right) \delta: 4.01$ (3H, s), 5.25 $(2 \mathrm{H}, \mathrm{s}), 7.44(6 \mathrm{H}, \mathrm{m}), 7.63(1 \mathrm{H}, \mathrm{s}), 10.42(1 \mathrm{H}, \mathrm{s})$.

5-Benzyloxy-4-methoxy-2-aminobenzaldehyde (21). Compound 21 was obtained from compound 20 as a yellow solid (64\% yield) as described for $\mathbf{6}$ and used directly to the next step without any purification; m.p. $180-184^{\circ} \mathrm{C}$. MS $m / z: 258.1[\mathrm{M}+\mathrm{H}]^{+}$.

6-Benzyloxy-7-methoxy-2-methylquinoline-3-carboxylic acid (22). Compound 22 was obtained from compound 21 as a greyish white solid (76\% yield) as described for 8; m.p. $274.2-278.4{ }^{\circ} \mathrm{C} .{ }^{1} \mathrm{H}-\mathrm{NMR}$ $\left(\mathrm{DMSO}-d_{6}\right) 2.82(3 \mathrm{H}, \mathrm{s}), 4.02(3 \mathrm{H}, \mathrm{s}), 5.27(2 \mathrm{H}, \mathrm{s}), 7.37-7.70(7 \mathrm{H}, \mathrm{m}), 8.66(1 \mathrm{H}, \mathrm{s})$.

\subsection{General Procedure for the Synthesis of Quinoline-3-carboxamide Derivatives 10-17, 23-27}

To a solution of 8, 9, $22(1 \mathrm{mmol})$ in dry DMF $(10 \mathrm{~mL})$ was added 1-hydroxybenzotrizole (HOBt) (1.5 mmol) and 1-ethyl-3-(3-dimethylaminopropyl)carbodiimide hydrochloride (EDC:HCl, $1.5 \mathrm{mmol})$. The mixture was stirred at room temperature for $2 \mathrm{~h}$, and then the corresponding amine $(2.0 \mathrm{mmol})$ and DIEA (2.0 eq) were added. The reaction mixture was stirred at room temperature for $5 \mathrm{~h}$, poured into 
ice water. The precipitate was filtered, washed with water, and then recrystallized with ethyl acetate or purified by column chromatography (silica gel) to give 10-17, 23-27.

N-Decyl-2-methyl-6-phenylquinoline-3-carboxamide (10). White solid, 84\% yield, m.p. 109.1-109.5 ${ }^{\circ} \mathrm{C} .{ }^{1} \mathrm{H}-\mathrm{NMR}$ (DMSO- $\left.d_{6}\right) \delta: 0.85(3 \mathrm{H}, \mathrm{t}), 1.25-1.32(14 \mathrm{H}, \mathrm{m}), 1.55(2 \mathrm{H}, \mathrm{t}), 2.68$ (3H, s), $3.28(2 \mathrm{H}, \mathrm{t}), 7.42(1 \mathrm{H}, \mathrm{t}, J=7.3 \mathrm{~Hz}), 7.53(2 \mathrm{H}, \mathrm{t}, J=7.2 \mathrm{~Hz}), 7.81(2 \mathrm{H}, \mathrm{d}, J=7.5 \mathrm{~Hz}), 8.02(1 \mathrm{H}, \mathrm{d}$, $J=8.8 \mathrm{~Hz}), 8.10\left(1 \mathrm{H}, \mathrm{dd}, J_{l}=8.8 \mathrm{~Hz}, J_{2}=2.0 \mathrm{~Hz}\right), 8.29(1 \mathrm{H}, \mathrm{d}, J=1.8 \mathrm{~Hz}), 8.33(1 \mathrm{H}, \mathrm{s}), 8.58(1 \mathrm{H}, \mathrm{s})$.

${ }^{13} \mathrm{C}-\mathrm{NMR}\left(\mathrm{DMSO}-d_{6}\right) \delta: 13.9,22.1,23.3,26.4,28.7,29.0,31.3,125.3,125.8,127.0,128.6,129.1$, 129.3, 131.4, 134.6, 137.7, 139.2, 146.3, 155.8, 167.7. HR-MS m/z: 403.2838 (calcd for $\mathrm{C}_{27} \mathrm{H}_{35} \mathrm{~N}_{2} \mathrm{O}$ $[\mathrm{M}+\mathrm{H}]^{+}:$403.2744). IR (KBr) cm $\mathrm{cm}^{-1}: 3230,3055,2917,2851,1634,1600,1578,837,755,699$.

N-Benzyl-2-methyl-6-phenylquinoline-3-carboxamide (11). White solid, 52\% yield, m.p. 168.9-171.1 ${ }^{\circ} \mathrm{C} .{ }^{1} \mathrm{H}-\mathrm{NMR}\left(\mathrm{DMSO}-d_{6}\right): 2.70(3 \mathrm{H}, \mathrm{s}), 4.53(2 \mathrm{H}, \mathrm{d}, J=5.8 \mathrm{~Hz}), 7.26-7.30(1 \mathrm{H}, \mathrm{m})$, $7.35-7.45(5 \mathrm{H}, \mathrm{m}), 7.54(2 \mathrm{H}, \mathrm{t}, J=7.3 \mathrm{~Hz}), 7.82(1 \mathrm{H}, \mathrm{d}, J=7.4 \mathrm{~Hz}), 8.03(1 \mathrm{H}, \mathrm{d}, J=8.7 \mathrm{~Hz}), 8.11$ $\left(1 \mathrm{H}, \mathrm{dd}, J_{1}=8.7 \mathrm{~Hz}, J_{2}=1.9 \mathrm{~Hz}\right), 8.33(1 \mathrm{H}, \mathrm{d}, J=1.7 \mathrm{~Hz}), 8.43(1 \mathrm{H}, \mathrm{s}), 9.17(1 \mathrm{H}, \mathrm{t}, J=5.9 \mathrm{~Hz})$. ${ }^{13} \mathrm{C}-\mathrm{NMR}\left(\mathrm{DMSO}-d_{6}\right.$ ) $\delta: 23.4,42.6,125.4,125.8,126.9,127.0,127.3,127.9,128.4,128.6,129.1$, $129.4,130.9,134.8,137.8,139.1,139.2,146.4,155.9,167.8$. HR-MS m/z: 353.1658 (calcd for $\mathrm{C}_{24} \mathrm{H}_{21} \mathrm{~N}_{2} \mathrm{O}[\mathrm{M}+\mathrm{H}]^{+}:$353.1648). IR (KBr) cm ${ }^{-1}: 3241,3066,1629,1598,1573,1023,838,755,699$.

N-Cyclopropyl-2-methyl-6-phenylquinoline-3-carboxamide (12). White solid, 22\% yield, m.p. 186.7-187.1 ${ }^{\circ} \mathrm{C} .{ }^{1} \mathrm{H}-\mathrm{NMR}\left(\mathrm{DMSO}-d_{6}\right) \quad \delta: 0.51-0.64(2 \mathrm{H}, \mathrm{m}), 0.69-0.75(2 \mathrm{H}, \mathrm{m}), 2.68$ (3H, s), 2.87-2.93 (1H, m), $7.42(1 \mathrm{H}, \mathrm{t}, J=7.4 \mathrm{~Hz}), 7.53(2 \mathrm{H}, \mathrm{t}, J=9.1 \mathrm{~Hz}), 7.81(2 \mathrm{H}, \mathrm{d}, J=7.4 \mathrm{~Hz}), 8.01(1 \mathrm{H}$, $\mathrm{d}, J=8.7 \mathrm{~Hz}), 8.10\left(1 \mathrm{H}, \mathrm{dd}, J_{1}=8.8 \mathrm{~Hz}, J_{2}=1.9 \mathrm{~Hz}\right), 8.28(1 \mathrm{H}, \mathrm{d}, J=1.7 \mathrm{~Hz}), 8.33(1 \mathrm{H}, \mathrm{s}), 8.65(1 \mathrm{H}, \mathrm{s})$. ${ }^{13}$ C-NMR (DMSO-d $\left.d_{6}\right) \delta: 5.7,22.8,23.3,125.3,125.7,127.0,127.8,128.6,129.1,129.4,131.0,134.7$, 137.8, 139.2, 146.3, 155.9, 168.9. HR-MS $m / z$ : 303.1564 (calcd for $\mathrm{C}_{20} \mathrm{H}_{19} \mathrm{~N}_{2} \mathrm{O}[\mathrm{M}+\mathrm{H}]^{+}:$303.1492). IR $(\mathrm{KBr}) \mathrm{cm}^{-1}:$ 3290, 3034, 1643, 1594, 1579, 1529, 696.

$N$-(2-Phenylethyl)-2-methyl-6-(p-tolyl)quinoline-3-carboxamide (13). Yellow solid, 64\% yield, m.p. 174.5-175.6 ${ }^{\circ} \mathrm{C} .{ }^{1} \mathrm{H}-\mathrm{NMR}$ (DMSO-d 6 ) $\delta: 2.37(3 \mathrm{H}, \mathrm{s}), 2.59(3 \mathrm{H}, \mathrm{s}), 2.89(2 \mathrm{H}, \mathrm{t}, J=7.1 \mathrm{~Hz}), 3.55(2 \mathrm{H}$, $\mathrm{t}, J=6.5 \mathrm{~Hz}), 7.22-7.35(7 \mathrm{H}, \mathrm{m}), 7.72(2 \mathrm{H}, \mathrm{d}, J=7.8 \mathrm{~Hz}), 7.99(1 \mathrm{H}, \mathrm{d}, J=8.7 \mathrm{~Hz}), 8.07(1 \mathrm{H}, \mathrm{d}$, $J=8.7 \mathrm{~Hz}), 8.22(1 \mathrm{H}, \mathrm{s}), 8.26(1 \mathrm{H}, \mathrm{s}), 8.68(1 \mathrm{H}, \mathrm{s}) .{ }^{13} \mathrm{C}-\mathrm{NMR}\left(\mathrm{DMSO}-d_{6}\right) \delta: 20.7,23.2,34.9,40.5$, 124.8, 125.8, 126.1 126.8, 128.3, 128.5, 128.7, 129.2, 129.7, 131.2, 134.5, 136.3, 137.3, 137.7, 139.3, 146.2, 155.7, 167.4. HR-MS m/z: 381.1971 (calcd for $\left.\mathrm{C}_{26} \mathrm{H}_{25} \mathrm{~N}_{2} \mathrm{O}[\mathrm{M}+\mathrm{H}]^{+}: 381.1961\right)$. IR (KBr) cm ${ }^{-1}$ : $3237,3063,3028,2936,1631,1599,1574,1491,811,748,700$.

N-(4-methoxyphenyl)-2-methyl-6-(p-tolyl) quinoline-3-carboxamide (14). Yellow solid, $84 \%$ yield, m.p. 221.0-221.8 ${ }^{\circ} \mathrm{C} .{ }^{1} \mathrm{H}-\mathrm{NMR}$ (DMSO-d $)$ $\delta: 2.37(3 \mathrm{H}, \mathrm{s}), 2.74(3 \mathrm{H}, \mathrm{s}), 3.76(3 \mathrm{H}, \mathrm{s}), 6.96(2 \mathrm{H}, \mathrm{d}$, $J=8.9 \mathrm{~Hz}), 7.34(2 \mathrm{H}, \mathrm{d}, J=8.0 \mathrm{~Hz}), 7.67-7.74(4 \mathrm{H}, \mathrm{m}), 8.04(1 \mathrm{H}, \mathrm{d}, J=8.7 \mathrm{~Hz}), 8.12(1 \mathrm{H}, \mathrm{dd}$, $\left.J_{l}=8.8 \mathrm{~Hz}, J_{2}=1.7 \mathrm{~Hz}\right), 8.31(1 \mathrm{H}, \mathrm{d}, J=1.3 \mathrm{~Hz}), 8.51(1 \mathrm{H}, \mathrm{s}), 10.49(1 \mathrm{H}, \mathrm{s}) .{ }^{13} \mathrm{C}-\mathrm{NMR}\left(\mathrm{DMSO}-d_{6}\right) \delta$ : 20.7, 23.4, 55.2, 113.9, 121.2, 124.9, 125.8, 126.8, 128.5, 129.5, 129.7, 131.1, 132.2, 135.1, 136.2, 137.3, 137.8, 146.3, 155.6, 166.0. HR-MS m/z: 383.1842 (calcd for $\mathrm{C}_{25} \mathrm{H}_{23} \mathrm{~N}_{2} \mathrm{O}_{2}[\mathrm{M}+\mathrm{H}]^{+}: 383.1754$ ). IR (KBr) cm ${ }^{-1}: 3247,3029,1648,1595,1525,1512,1245,813$. 
N-Benzyl-2-methyl-6-(p-tolyl)quinoline-3-carboxamide (15). Yellow solid, $31 \%$ yield, m.p. 181.8-182.4 ${ }^{\circ} \mathrm{C} .{ }^{1} \mathrm{H}-\mathrm{NMR}\left(\mathrm{DMSO}-d_{6}\right) \delta: 2.31(3 \mathrm{H}, \mathrm{s}), 2.69(3 \mathrm{H}, \mathrm{s}), 4.53(2 \mathrm{H}, \mathrm{d}), 7.46-7.24(7 \mathrm{H}, \mathrm{m})$, $7.73(2 \mathrm{H}, \mathrm{d}, J=7.9 \mathrm{~Hz}), 8.01(1 \mathrm{H}, \mathrm{d}, J=8.7 \mathrm{~Hz}), 8.08(1 \mathrm{H}, \mathrm{d}, J=9.1 \mathrm{~Hz}), 8.29(1 \mathrm{H}, \mathrm{s}), 8.41(1 \mathrm{H}, \mathrm{s})$, $9.15(1 \mathrm{H}, \mathrm{m}) .{ }^{13} \mathrm{C}-\mathrm{NMR}\left(\mathrm{DMSO}-d_{6}\right) \delta: 20.7,23.4,42.6,124.9,125.8,126.8,126.9,127.3,128.4$, $128.5,129.3,129.7,130.9,134.8,136.3,137.3,137.7,139.2,146.3,155.7,167.9$. HR-MS $\mathrm{m} / z$ : 367.1892 (calcd for $\mathrm{C}_{25} \mathrm{H}_{23} \mathrm{~N}_{2} \mathrm{O}[\mathrm{M}+\mathrm{H}]^{+}:$367.1805). IR (KBr) cm ${ }^{-1}: 3273,2919,1631,1594,1540,808$.

N-Cyclopropyl-2-methyl-6-(p-tolyl) quinoline-3-carboxamide (16). White solid, 43\% yield, m.p. 220.0-220.9 ${ }^{\circ} \mathrm{C} .{ }^{1} \mathrm{H}-\mathrm{NMR}\left(\mathrm{DMSO}-d_{6}\right) \delta: 0.58(2 \mathrm{H}, \mathrm{s}), 0.73(2 \mathrm{H}, \mathrm{s}), 2.37(3 \mathrm{H}, \mathrm{s}), 2.67$ (3H, s), 2.88 $(1 \mathrm{H}, \mathrm{s}), 7.34(2 \mathrm{H}, \mathrm{d}, J=5.6 \mathrm{~Hz}), 7.71(2 \mathrm{H}, \mathrm{d}, J=5.4 \mathrm{~Hz}), 8.01(1 \mathrm{H}, \mathrm{s}), 8.06(1 \mathrm{H}, \mathrm{s}), 8.24(1 \mathrm{H}, \mathrm{s}) 8.30$ $(1 \mathrm{H}, \mathrm{s}), 8.62(1 \mathrm{H}, \mathrm{s}) .{ }^{13} \mathrm{C}-\mathrm{NMR}\left(\mathrm{DMSO}-d_{6}\right) \delta: 20.7,23.3,23.5,32.2,40.0,50.8,124.8,125.9,126.8$, $128.5,129.1,129.7,131.4,134.5,136.3,137.2,137.6,146.2,155.7,167.3$. HR-MS m/z: 317.1633 (calcd for $\mathrm{C}_{21} \mathrm{H}_{21} \mathrm{~N}_{2} \mathrm{O}[\mathrm{M}+\mathrm{H}]^{+}:$317.1648). IR (KBr) cm ${ }^{-1}: 3279,2962,2869,1632,1542,806$.

N-(tert-Butyl)-2-methyl-6-(p-tolyl)quinoline-3-carboxamide (17). White solid, 24\% yield, m.p. 181.7-183.6 ${ }^{\circ} \mathrm{C} .{ }^{1} \mathrm{H}-\mathrm{NMR}\left(\mathrm{CDCl}_{3}\right) \delta: 1.47(9 \mathrm{H}, \mathrm{s}), 2.36(3 \mathrm{H}, \mathrm{s}), 2.78(3 \mathrm{H}, \mathrm{s}), 7.23(2 \mathrm{H}, \mathrm{d}, J=8.0 \mathrm{~Hz})$, $7.51(2 \mathrm{H}, \mathrm{d}, J=8.1 \mathrm{~Hz}), 7.89(2 \mathrm{H}, \mathrm{d}, J=9.4 \mathrm{~Hz}), 8.01(1 \mathrm{H}, \mathrm{d}, J=9.3 \mathrm{~Hz}), 8.08(1 \mathrm{H}, \mathrm{s}) .{ }^{13} \mathrm{C}-\mathrm{NMR}$ $\left(\mathrm{DMSO}-d_{6}\right) \delta: 20.7,23.1,28.5,50.9,124.8,125.9,126.7,128.5,128.9,129.7,132.3,134.1,136.3$, 137.2, 137.5, 146.1, 155.5, 167.7. HR-MS $m / z$ : 333.2041 (calcd for $\mathrm{C}_{22} \mathrm{H}_{25} \mathrm{~N}_{2} \mathrm{O}[\mathrm{M}+\mathrm{H}]^{+}: 333.1961$ ). IR $(\mathrm{KBr}) \mathrm{cm}^{-1}: 3252,2967,1647,1600,1548,929,813,802$.

6-(Benzyloxy)-7-methoxy-2-methyl-N-(benzyl)quinoline-3-carboxamide (23). White solid, 33\% yield, m.p. 214.6-215.3 ${ }^{\circ} \mathrm{C} .{ }^{1} \mathrm{H}-\mathrm{NMR}$ (DMSO- $\left.d_{6}\right) \delta: 2.60(3 \mathrm{H}, \mathrm{s}), 3.91(3 \mathrm{H}, \mathrm{s}), 4.46(2 \mathrm{H}, \mathrm{d}, J=5.7 \mathrm{~Hz}), 5.19$ $(2 \mathrm{H}, \mathrm{s}), 7.25(1 \mathrm{H}, \mathrm{s}), 7.33-7.41(8 \mathrm{H}, \mathrm{m}), 7.46-7.49(3 \mathrm{H}, \mathrm{m}), 8.12(1 \mathrm{H}, \mathrm{s}), 9.00(1 \mathrm{H}, \mathrm{d}, J=7.1 \mathrm{~Hz})$. ${ }^{13} \mathrm{C}-\mathrm{NMR}\left(\mathrm{DMSO}-d_{6}\right) \delta: 23.2,42.5,55.7,69.9,107.1,107.2,120.6,126.8,127.2,128.0,128.3,128.5$, 133.1, 136.5, 139.4, 144.3, 148.2, 153.0, 153.2, 168.2. HR-MS m/z: 413.1870 (calcd for $\mathrm{C}_{26} \mathrm{H}_{25} \mathrm{~N}_{2} \mathrm{O}_{3}$ $[\mathrm{M}+\mathrm{H}]^{+}:$413.1860). IR (KBr) $\mathrm{cm}^{-1}:$ 3283, 3029, 2964, 2935, 1634, 1540, 748, 703.

6-(Benzyloxy)-7-methoxy-2-methyl-N-(4-methylphenyl)quinoline-3-carboxamide (24). White solid, $44 \%$ yield, m.p. $202.1-202.8{ }^{\circ} \mathrm{C} .{ }^{1} \mathrm{H}-\mathrm{NMR}$ (DMSO- $\left.d_{6}\right) \delta: 2.28(3 \mathrm{H}, \mathrm{s}), 2.93(3 \mathrm{H}, \mathrm{s}), 4.02(3 \mathrm{H}, \mathrm{s}), 5.28$ $(2 \mathrm{H}, \mathrm{s}), 7.16-7.81(11 \mathrm{H}, \mathrm{m}), 8.93(1 \mathrm{H}, \mathrm{s}), 10.78(1 \mathrm{H}, \mathrm{s}) .{ }^{13} \mathrm{C}-\mathrm{NMR}$ (DMSO- $\left.d_{6}\right) \delta: 23.4,55.2,113.9$, 121.2, 125.4, 125.7, 127.0, 127.9, 128.7, 129.1, 129.6, 131.1, 132.2, 135.1, 137.9, 139.2, 146.4, 155.6, 155.8, 166.0. HR-MS $m / z: 413.1869$ (calcd for $\mathrm{C}_{26} \mathrm{H}_{25} \mathrm{~N}_{2} \mathrm{O}_{3}[\mathrm{M}+\mathrm{H}]^{+}: 413.1860$ ). IR (KBr) cm ${ }^{-1}: 3261$, $3033,2927,1648,1522,752$.

6-Benzyloxy-7-methoxy-2-methyl-N-(2-(thiophen-2-yl)ethyl)quinoline-3-carboxamide (25). White solid, $27 \%$ yield, m.p. $178.8-179.5{ }^{\circ} \mathrm{C} .{ }^{1} \mathrm{H}-\mathrm{NMR}$ (DMSO- $\left.d_{6}\right) \delta: 2.58(3 \mathrm{H}, \mathrm{s}), 3.09(2 \mathrm{H}, \mathrm{t}), 3.48-3.57(2 \mathrm{H}, \mathrm{q})$, $3.93(3 \mathrm{H}, \mathrm{s}), 5.22(2 \mathrm{H}, \mathrm{s}), 6.93-7.01(2 \mathrm{H}, \mathrm{m}), 7.33-7.46(6 \mathrm{H}, \mathrm{m}), 7.48-7.55(2 \mathrm{H}, \mathrm{m}), 8.05(1 \mathrm{H}, \mathrm{s})$, $8.63(1 \mathrm{H}, \mathrm{s}) .{ }^{13} \mathrm{C}-\mathrm{NMR}\left(\mathrm{DMSO}-d_{6}\right) \delta: 23.1,29.2,40.7,55.7,69.9,107.1,107.2,120.5,124.1,125.3$, 126.9, 128.0, 128.1, 128.4, 128.5, 132.9, 136.5, 141.5, 144.2, 148.2, 152.9, 153.2, 168.2. HR-MS m/z: 433.1681 (calcd for $\mathrm{C}_{25} \mathrm{H}_{25} \mathrm{O}_{3} \mathrm{~N}_{2} \mathrm{~S}[\mathrm{M}+\mathrm{H}]^{+}:$433.1580). IR (KBr) $\mathrm{cm}^{-1}$ : 3311, 3081, 2966, 2924, 2863, $1636,1536$. 
6-Benzyloxy-7-methoxy-2-methyl-N-(tert-butyl)quinoline-3-carboxamide (26). White solid, 35\% yield, m.p. 136.8-138.1 ${ }^{\circ} \mathrm{C} .{ }^{1} \mathrm{H}-\mathrm{NMR}$ (DMSO- $\left.d_{6}\right) \delta: 1.39$ (9H, s). $2.59(3 \mathrm{H}, \mathrm{s}), 3.93(3 \mathrm{H}, \mathrm{s}), 5.20$ (2H, s), 7.33-7.52 (7H, m), $7.99(1 \mathrm{H}, \mathrm{s}), 8.06(1 \mathrm{H}, \mathrm{s}) .{ }^{13} \mathrm{C}-\mathrm{NMR}$ (DMSO-d $)$ o: 22.8, 28.5, 50.8, 55.7, 69.7, $107.1,120.6,128.0,128.5,132.6,136.5,143.9,148.0,152.7,152.9,168.1$. HR-MS m/z: 379.2025 (calcd for $\mathrm{C}_{23} \mathrm{H}_{27} \mathrm{~N}_{2} \mathrm{O}_{3}[\mathrm{M}+\mathrm{H}]^{+}$: 379.2016). IR (KBr) cm ${ }^{-1}$ : 3227, 3029, 2965, 1666, 1604, 1503, 748.

6-Benzyloxy-7-methoxy-2-methyl-N-(n-pentyl)quinoline-3-carboxamide (27). Yellow solid, 73\% yield, m.p. $168.0-168.5{ }^{\circ} \mathrm{C} .{ }^{1} \mathrm{H}-\mathrm{NMR}$ (DMSO- $\left.d_{6}\right) \delta: 0.89(3 \mathrm{H}, \mathrm{t}, J=6.6 \mathrm{~Hz}), 1.33-1.34(4 \mathrm{H}, \mathrm{m}), 1.51-1.56$ $(2 \mathrm{H}, \mathrm{m}), 2.61(3 \mathrm{H}, \mathrm{s}), 3.24-3.33(2 \mathrm{H}, \mathrm{m}), 3.93(3 \mathrm{H}, \mathrm{s}), 5.21(2 \mathrm{H}, \mathrm{s}), 7.35-7.53(7 \mathrm{H}, \mathrm{s}), 8.05(1 \mathrm{H}, \mathrm{s})$, $8.45(1 \mathrm{H}, \mathrm{t}, J=5.3 \mathrm{~Hz}) .{ }^{13} \mathrm{C}-\mathrm{NMR}\left(\mathrm{DMSO}-d_{6}\right) \delta: 13.9,21.8,23.0,28.6,28.7,55.7,69.9,107.1,107.2$, 120.6, 128.0, 128.5, 128.8, 132.8, 136.5, 144.1, 148.1, 152.9, 153.1, 168.1. HR-MS m/z: 393.2183 (calcd for $\mathrm{C}_{24} \mathrm{H}_{29} \mathrm{~N}_{2} \mathrm{O}_{3}[\mathrm{M}+\mathrm{H}]^{+}:$393.2173). IR (KBr) cm $\mathrm{cm}^{-1}$ : 3283, 2929, 2857, 1634, 1500.

\subsection{CETP Inhibition Assay}

CETP activity was determined by detecting the exchange of radioactive cholesteryl ester between labelled HDL and unlabelled LDL [18]. Briefly, fresh rabbit serum containing CETP was incubated in TSE buffer ( $50 \mathrm{mM}$ Tris, $50 \mathrm{mM} \mathrm{NaCl}, 2 \mathrm{mM}$ EDTA and 1\% bovine serum albumin) containing $10 \mu \mathrm{mol} / \mathrm{L}$ test compounds dissolved in dimethyl sulfoxide [final concentration of both rabbit serum and dimethyl sulfoxide in incubation mixture was $0.5 \%$ in $300 \mu \mathrm{L}$ volume] for $4 \mathrm{~h}$ at $37{ }^{\circ} \mathrm{C}$. Then incubated for another $16 \mathrm{~h}$ at $37{ }^{\circ} \mathrm{C}$ with $0.5 \mu \mathrm{L}\left[{ }^{3} \mathrm{H}\right]$ cholesteryl ester-labeled HDL and $5 \mu \mathrm{L}$ unlabelled LDL in $600 \mu \mathrm{L}$ volume. LDL was precipitated with dextran sulfate (final concentration: $0.027 \%$ ) and $\mathrm{MgCl}_{2}$ (final concentration: $27 \mathrm{mM}$ ) for $30 \mathrm{~min}$. Centrifuged at 5,000 $\mathrm{g}$ and $4{ }^{\circ} \mathrm{C}$ for $30 \mathrm{~min}$ to get the supernatant, and its radioactivity was measured in a liquid scintillation counter (Wallac 1410, Pharmacia, Uppsala, Sweden). The CETP activity was determined by the decrease in radioactivity versus that of a blank without serum.

\section{Conclusions}

In conclusion, a series of novel quinoline-3-carboxamide derivatives 10-17 and 23-27 were synthesized and their CETP inhibitory activity evaluated. Generally, compounds substituted with 6-benzyloxy-7-methoxy groups possessed more potent CETP inhibitory activity. Compounds $\mathbf{2 4}$ and 26 exhibited promising inhibitory activity of CETP (80.1\%) compared with the lead compound $\mathbf{1}$ (30\%). As a novel CETP inhibitor scaffold, further structural modifications of the quinoline-3-carboxamide moiety are progressing in our lab.

\section{Acknowledgments}

The authors thank Yi-Ping Wang of the Shanghai Insititute of Materia Medica, Chinses Academy of Science for the activity assays. 


\section{References and Notes}

1. Bays, H.; Stein, E.A. Pharmacotherapy for dyslipidaemia-Current therapies and future agents. Expert. Opin. Pharmacother. 2003, 4, 1901-1938.

2. Lange, R.A.; Lindsey, M.L. HDL-cholesterol levels and cardiovascular risk: acCETPing the context. Eur. Heart. J. 2008, 29, 2708-2709.

3. Assmann, G.; Nofer, J.R. Atheroprotective effects of high-density lipoproteins. Ann. Rev. Med. 2003, 54, 321-341.

4. Besler, C.; Heinrich, K.; Riwanto, M.; Luescher, T.F.; Landmesser, U. High-density lipoprotein-mediated anti-atherosclerotic and endothelial-protective effects: A potential novel therapeutic target in cardiovascular disease. Curr. Pharm. Des. 2010, 16, 1480-1493.

5. Tall, A.R. Plasma cholesteryl ester transfer protein. J. Lipid. Res. 1993, 34, 1255-1274.

6. Doggrell, S.A. Inhibitors of cholesteryl ester transfer protein-A new approach to coronary artery disease. Expert. Opin. Investig. Drugs. 2006, 15, 99-106.

7. Mabuchi, H.; Inazu, A. Cholesteryl ester transfer protein inhibitors: New strategies for raising high-density lipoprotein cholesterol. Future Lipidol. 2006, 1, 487-500.

8. Sikorski, J.A. Cholesteryl ester transfer protein inhibitors as potential new therapies for coronary artery disease. Expert. Opin. Ther. Pat. 2006, 16, 753-772.

9. Clark, R.W. Raising high-density lipoprotein with chollesteryll ester transfer protein inhibitors. Curr. Opin. Pharmacol. 2006, 6, 162-168.

10. Barter, P.J.; Kastelein, J.J.P. Targeting cholesteryl ester transfer protein for the prevention and management of cardiovascular disease. J. Am. Coll. Cardiol. 2006, 47, 492-499.

11. Cannon, C.P.; Shah, S.; Dansky, H.M.; Davidson, M.; Brinton, E.A.; Gotto, A.M.; Stepanavage, M.; Liu, S.X.; Gibbons, P.; Ashraf, T.B.; et al. Safety of anacetrapib in patients with or at high risk for coronary heart disease. N. Engl. J. Med. 2010, 25, 2406-2415.

12. Stein, E.A.; Stroes, E.S.G.; Steiner, G.; Buckley, B.M.; Capponi, A.M.; Burgess, T.; Niesor, E.J.; Kallend, D.; Kastelein, J.J.P. Safety and tolerability of dalcetrapib. Am. J. Cardio. 2009, 1, 82-91.

13. Nicholls, S.J.; Brewer, H.B.; Kastelein, J.J.P.; Krueger, K.A.; Wang, M.D.; Shao, M.; Hu, B.; McErlean, E.; Nissen, S.E. Effects of the CETP inhibitor evacetrapib administered as monotherapy or in combination with statins on HDL and LDL cholesterol a randomized controlled trial. JAMA 2011, 19, 2099-2109.

14. Xiong, X.Q.; Zhao, D.M.; Bu, P.F.; Liu, Y.; Ren, J.H.; Wang, J.; Cheng, M.S. CoMFA, CoMSIA and eigenvalue analysis on dibenzodioxepinone and dibenzodioxocinone derivatives as cholesteryl ester transfer protein inhibitors. Molecules 2008, 13, 1822-1839.

15. Qiu, X.; Mistry, A.; Ammirati, M.J.; Chrunyk, B.A.; Clark, R.W.; Cong, Y.; Culp, J.S.; Danley, D.E.; Freeman, T.B.; Geoghegan, K.F.; et al. Crystal structure of cholesteryl ester transfer protein reveals a long tunnel and four bound lipid molecules. Nat. Struct. Mol. Biol. 2007, 2, 106-113.

16. Patteux, C.; Foucout, L.; Bohn, P.; Dupas, G.; Leprince, J.; Tonon, M.C.; Dehouck, B.; Marsais, F.; Papamicael, C.; Levacher, V. Solid phase synthesis of a redox delivery system with the aim of targeting peptides into the brain. Org. Biomol. Chem. 2006, 4, 817-825.

17. Kodama, S.; Takita, H.; Kajimoto, T.; Nishide, K.; Node, M. Synthesis of Amaryllidaceae alkaloids, siculine, oxocrinine, epicrinine, and buflavine. Tetrahedron 2004, 60, 4901-4907. 
18. Okamoto, H.; Iwamoto, Y.; Maki, M.; Sotani, T.; Yonemori, F.; Wakitani, K. Effect of JTT-705 on cholesteryl ester transfer protein and plasma lipid levels in normolipidemic animals. Eur. J. Pharmacol. 2003, 466, 147-154.

Sample Availability: Samples of the compounds 10-17, 23-27 are available from the authors.

(C) 2012 by the authors; licensee MDPI, Basel, Switzerland. This article is an open access article distributed under the terms and conditions of the Creative Commons Attribution license (http://creativecommons.org/licenses/by/3.0/). 